\title{
HIGH THERMAL CONDUCTIVITY CRYOGENIC RF FEEDTHROUGHS FOR HIGHER ORDER MODE COUPLERS *
}

\author{
C. E. Reece", E. F. Daly, T. Elliott, H. L. Phillips, J.P Ozelis, T. Rothgeb, K. Wilson, and G. Wu \\ Jefferson Lab, 12000 Jefferson Avenue, Newport News, VA 23606 U.S.A.
}

\begin{abstract}
The use of higher-order-mode (HOM) pickup probes in the presence of significant fundamental RF fields can present a thermal challenge for $\mathrm{CW}$ or high average power SRF cavity applications. The electric field probes on the HOM-damping couplers on the JLab "High Gradient" (HG) and "Low Loss" (LL) seven-cell cavities for the CEBAF upgrade are exposed to approximately $10 \%$ of the peak magnetic field in the cavity. To avoid significant dissipative losses, these probes must remain superconducting during operation. Typical cryogenic rf feedthroughs provide a poor thermal conduction path for the probes and provide inadequate stabilization. We have developed solutions that meet the requirements, providing a direct thermal path from the niobium probe, thorough single-crystal sapphire, to bulk copper which can be thermally anchored. Designs, electromagnetic and thermal analyses, and performance data will be presented.
\end{abstract}

\section{HOM COUPLER REQUIREMENTS}

The improved cavity designs developed and considered for use in the CEBAF $12 \mathrm{GeV}$ Upgrade project have two HOM couplers on each end.[1] These couplers are particularly required to dampen dipole modes that would contribute to beam breakup (BBU). The design beam requirements for the Upgrade is $460 \mu \mathrm{A} \mathrm{CW}$.[2] Damping of key modes to $Q_{1}<10^{7}$ is sufficient. The analyzed BBU safe criterion is $Z=(R / Q) * Q_{1}<6.3 * 10^{8} \Omega / \mathrm{cm}^{2}$.[3]

The coupler used is an adaptation of the DESY/Saclay HOM coupler used in TTF. Its function depends on resonant rejection of the fundamental such that the $Q$ external of the fundamental is $>3 \times 10^{11}$ while damping the HOMs. Therefore, although the coupler extracts very little fundamental power, the amplitude of fundamental fields present within the coupler may be quite high. Because the iris diameters of the $\mathrm{HG}$ and LL cavity designs are smaller than for the former CEBAF cell shape (OC), the HOM couplers were moved as close to end cells as practical in HG and LL in order to attain maximum HOM damping. Maintaining thermal stability under this condition presents the problem at hand.

3-D modeling of the fundamental fields in the couplers adjoining a single-cell LL cavity was undertaken[4] to establish the relative amplitude of the magnetic fields and their distribution on the relatively isolated pick-up probe. The tip region of the pickup probe is found to experience $\sim 10 \%$ of $H_{\max }$ in the cell. It is thus clear that to avoid rf dissipative losses that dominate all others, the probe must

\footnotetext{
* This manuscript has been authorized by SURA, Inc. under Contract No. DE-AC05-84ER-40150 with the U.S. Department of Energy. ${ }^{\#}$ reece $(a$, ilab.org
}

be superconducting and thermally stabilized. Thermal stabilization of the probe must be through the rf feedthrough. Figure 1 shows the configuration of two couplers at the waveguide fundamental power coupler (FPC) end of each cavity.

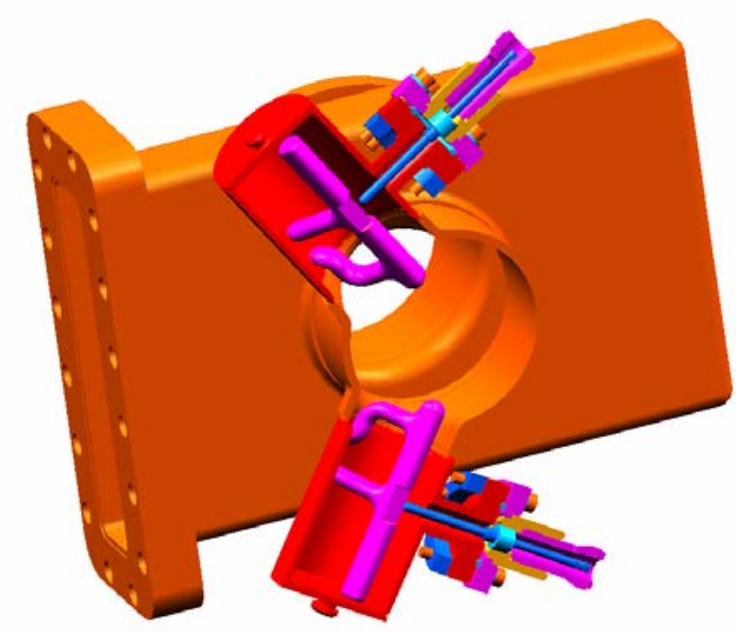

Figure 1. Cross-section of two HOM couplers between the FPC and cells of the HG and LL cavities.

\section{ANALYSIS OF NB PROBE HEATING}

The probe tip of a DESY-type High Order Mode (HOM) coupler is located right in the electric field minimum for the cavity fundamental mode to reduce the power transmission through the HOM coupler. This electric field minimum location is also a magnetic field maximum in this transmission line type HOM coupler. The current on the coupler tip surface causes the heating problem. To maximize the HOM damping of CEBAF Upgrade cavities both on LL and HG shapes, the HOM coupler position was moved closer to the end cell. This increased the magnetic field ratio between the coupler tip to the cavity equator and produced extra heat on the coupler tip. If the heat is not sufficiently conducted away through the probe feedthrough, the tip may become normal conducting, leading to a thermal run-away that dramatically lowers the cavity quality factor $(Q)$.

A 3-D RF model of the coupler/cavity system was constructed in HFSS ${ }^{\mathrm{TM}}$. Sensitivity analyses were made of the heat dissipated in the probe under a variety of conditions, including three variations of the shape of the probe tip. Figure 2 shows the magnetic field strength distribution in two cases. Scaling the field amplitudes to correspond to operational conditions of $E_{\text {acc }}=20 \mathrm{MV} / \mathrm{m}$, 


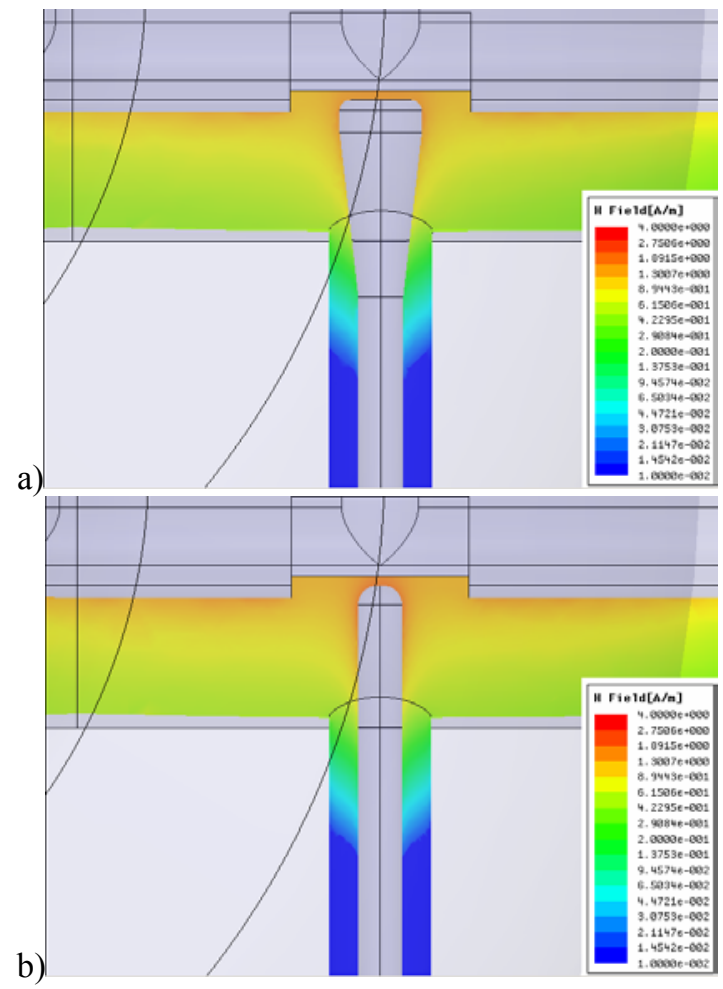

Figure 2 The local magnetic field around probe Tip 1 (a), and Tip 2(b).

assuming BCS surface impedance, and integrating square of the surface magnetic field, we calculate the heat into the probe. See Figure 3.

$$
W_{\text {loss-on-probe }}=\frac{1}{2} R_{s(B C S)}\left(\int_{\text {total }}\left|H_{\perp}\right|^{2} d s\right)
$$

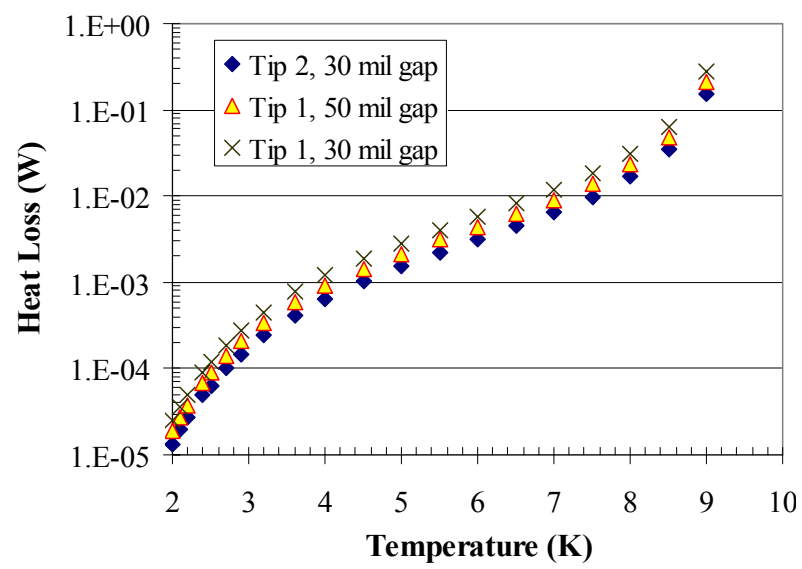

Figure 3. Heat load on $\mathrm{Nb}$ probe in HG \& LL cavity HOM coupler at $20 \mathrm{MV} / \mathrm{m} \mathrm{CW}$, assuming $\mathrm{Nb} \mathrm{BCS}$ surface impedance at $1.5 \mathrm{GHz}$.

Among the options modeled, as expected, the smaller tip produces less heating. The thermally more conservative "Tip 2" geometry was selected for use in cavities of the Renascence cryomodule. This choice reduced the HOM damping, but still comfortably meets the specifications for the $12 \mathrm{GeV}$ upgrade project. Also clearly illustrated is the need to thermally stabilize the probe. Within the range of geometries modeled, one may summarize the BCS heat generated on a niobium probe at $20 \mathrm{MV} / \mathrm{m}$ as 3-6 mW @ $6 \mathrm{~K}$ and 16-30 mW @ 8 K. This power must be sunk to $2 \mathrm{~K}$ through the feedthrough dielectric to the flange and cavity beampipe or via supplemental strapping to the feedthrough body.

The probe geometry producing the lower end of the ranges was used on the feedthroughs in the cavities of the Renascence cryomodule.

\section{RF FEEDTHROUGH DESIGNS}

Three types of RF feedthroughs have been examined for use in these HOM couplers. Before we recognized the criticality of the thermal conduction, we attempted to use the Kyocera feedthroughs that have functioned successfully on TTF and SNS cryomodules. These applications are pulsed rather than $\mathrm{CW}$. Initial tests on the prototype HG cavity showed significant heating and long thermal time constants, particularly when copper probes were used.[5] The thermal conductivity of the sealing dielectric was too low for our application.

JLab in partnership with Ceramaseal ${ }^{\circledR}$ undertook the development of a new cryogenic RF feedthrough that incorporated a high-purity alumina dielectric.[6] These feedthroughs were successfully produced, tested, and judged acceptable for the CEBAF upgrade application.

In addition, JLab undertook a parallel development of a second new RF feedthrough design that was more tightly specialized to our application. The concept was to directly braze the niobium probe to a single-crystal sapphire dielectric and the sapphire to a substantial copper sleeve captured in the stainless steel mounting flange. The copper sleeve is available externally for thermal strapping, if needed. Otherwise, the feedthrough is conduction cooled through the flange, gasket and bolt hardware. A deliberate compromise was made to sacrifice broadband VSWR for generous thermal conduction.

To take advantage of the excellent compressive strength of sapphire, the design gives both braze joints to the dielectric a cylindrical capture geometry. The Inconel ${ }^{\mathrm{TM}}$ center conductor from the Type-N connector is also brazed directly to the niobium probe, threading the sapphire center without a bond.

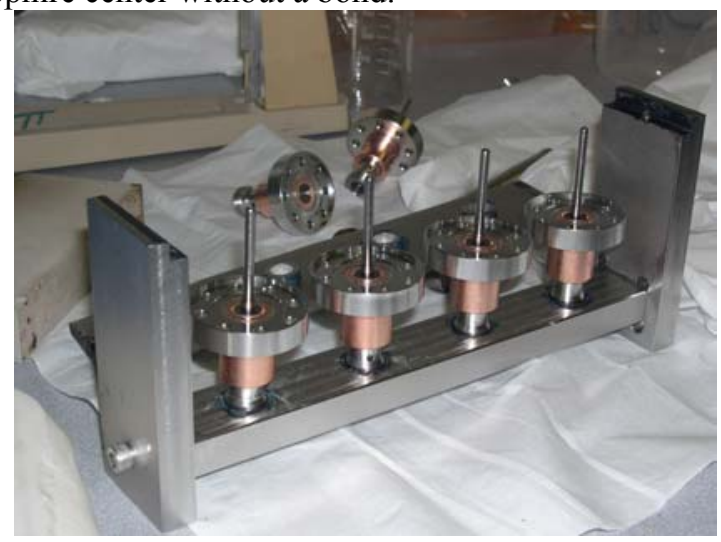

Figure 4. JLab single-crystal sapphire RF feedthroughs. 
32 RF feedthroughs of the latter type were fabricated and tested at JLab and assembled into the Renascence cavity string. Figure 4 shows four such assemblies in a braze fixture.

\section{THERMAL ANALYSIS AND EXPERITMENTAL TESTING}

The thermal conduction properties of each of three types of RF feedthroughs have been characterized by a combination of finite element analyses with temperature dependent material parameters and instrumented thermal conduction measurements. These simulations and measurements sought to determine the temperature of the tip of the niobium probe given a particular input heat load at the tip and a representative cooling boundary condition at the feedthrough flange. Table 1 summarizes the results.

The design using a sapphire dielectric provides a superior thermal bond between the niobium probe and the mounting flange. Analysis indicates that with the external copper collar stabilized at $3 \mathrm{~K}$, a heat flux of more than $100 \mathrm{~mW}$ could be stably sustained a keep the probe below the critical temperature of niobium.

Figure 5 shows the results of two thermal models that keep the probe tip $<7 \mathrm{~K}$. Figure 6 presents measurements on two feedthrough designs of probe tip and flange temperatures in a test fixture. The data demonstrate very good thermal conduction through the RF feedthrough.

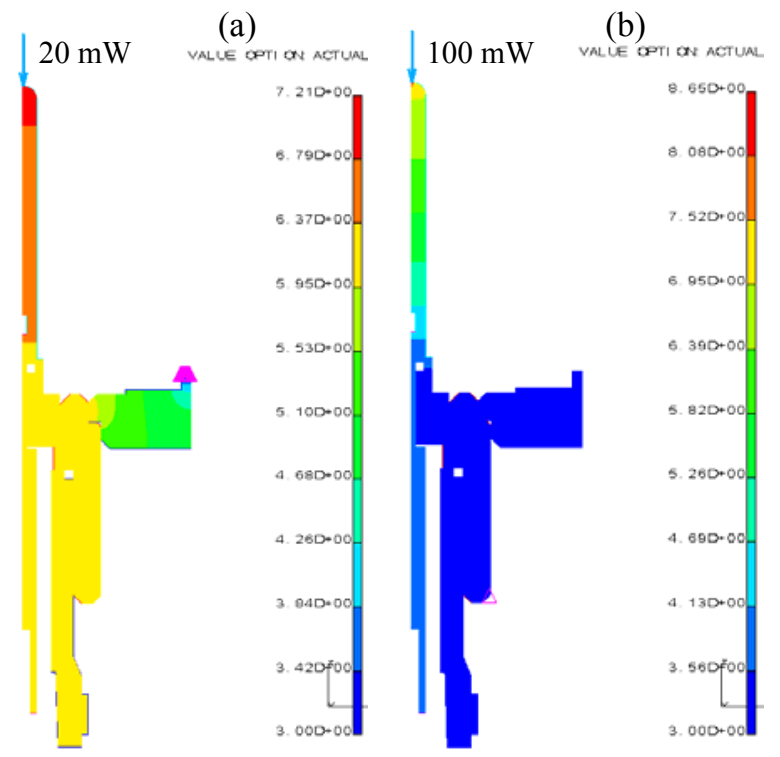

Figure 5. FEA thermal model of the sapphire-dielectric $\mathrm{RF}$ feedthrough with a) $20 \mathrm{~mW}$ load to tip and $3 \mathrm{~K}$ flange, and b) $100 \mathrm{~mW}$ load to the tip with $3 \mathrm{~K}$ copper collar. In both cases, the probe tip stabilizes at $6.9 \mathrm{~K}$.

\section{CONCLUSION}

Thermally stabilized cryogenic RF feedthroughs suitable for use in high field CW applications - such as some HOM coupler designs in superconducting accelerating cavities - have been developed and tested. The present designs are suitable for use on the CEBAF
$12 \mathrm{GeV}$ upgrade cavities. Other high-gradient $\mathrm{CW}$ applications may also benefit from this development.

\section{ACKNOWLEGEMENTS}

The authors wish to acknowledge the vital contributions to this work by R. Sarno, of CeramTec North America Corp., Ceramaseal Division, and B. Manus, J. Henry, and S. Dutton of JLab.

Table 1: Niobium probe tip temperature with three RF feedthrough designs conduction cooled through their stainless steel flange.

\begin{tabular}{|c|c|c|c|}
\hline $\begin{array}{c}\text { RF Feedthrough } \\
\text { Design }\end{array}$ & $\begin{array}{c}\mathrm{T}_{\text {tip }} @ \\
10 \mathrm{~mW}\end{array}$ & $\begin{array}{c}\mathrm{T}_{\text {tip }} @ \\
20 \mathrm{~mW}\end{array}$ & $\begin{array}{c}\text { Suitability for } \\
12 \mathrm{GeV} \\
\text { CEBAF }\end{array}$ \\
\hline $\begin{array}{c}\text { Kyocera design } \\
\text { used on TTF and } \\
\text { SNS (pulsed RF) }\end{array}$ & $>13 \mathrm{~K}$ & $16 \mathrm{~K}$ & Not viable \\
\hline $\begin{array}{c}\text { JLab/Ceramaseal } \\
\text { design }\end{array}$ & $5.5 \mathrm{~K}$ & $<9.2 \mathrm{~K}$ & $\begin{array}{c}\text { Acceptable } \\
\text { and } \\
\text { demonstrated }\end{array}$ \\
\hline $\begin{array}{c}\text { JLab sapphire- } \\
\text { dielectric design }\end{array}$ & $<5 \mathrm{~K}$ & $\sim 6.9 \mathrm{~K}$ & $\begin{array}{c}\text { Confidently } \\
\text { better }\end{array}$ \\
\hline
\end{tabular}

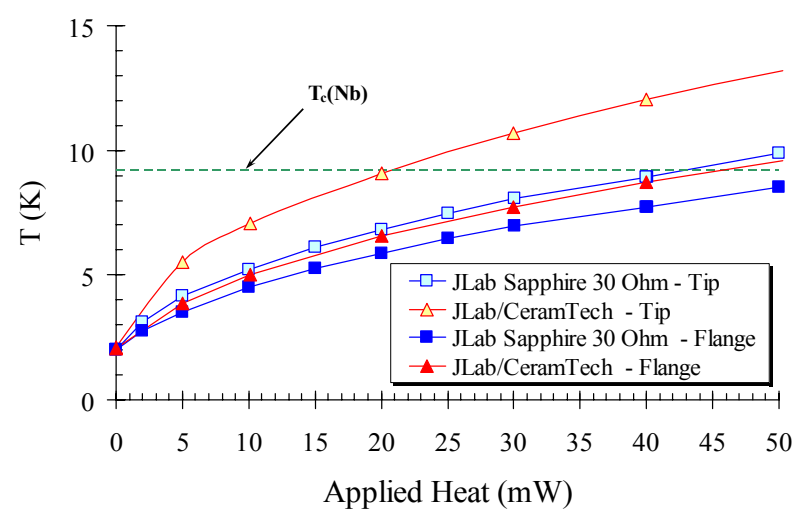

Figure 6. Flange and probe tip temperatures as a function of heat applied to the probe tip, two designs.

\section{REFERENCES}

[1] J. Sekutowicz et al., "Cavities for JLab's $12 \mathrm{GeV}$ Upgrade,' Proc. PAC 2003, TPZB085.

[2] L. Harwood, "JLab 12-GeV Upgrade," contribution to this conference: WOAD004.

[3] B. Yunn, "HOM damping Requirement for $12 \mathrm{GeV}$ Upgrade," JLab Technote JLAB-TN-04-035, Nov. 2004.

[4] G. Wu et al., "Evaluation of HOM Coupler Probe Heating by HFSSTM Simulation," JLab Technote JLAB-TN-04-027, Sept. 2004.

[5] P. Kneisel et al., "First Cryogenic Tests with JLab's New Upgrade Cavities,” Proc. LINAC2004 MOP84.

[6] CeramTec North America Corp., Ceramaseal Division, New Lebanon, NY. PN: 20248. 